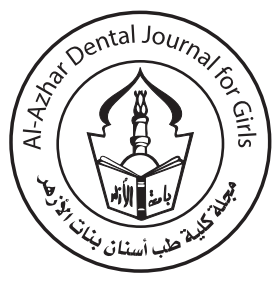

\title{
The Effect of Different Occlusal Concepts on Masticatory Efficiency in Implant Supported Mandibular Over-dentures
}

\author{
Hager F. El-sadany ${ }^{1 *}$, Amany A. Abd El-fattah ${ }^{2}$, Nesreen A. El-Mahrouky ${ }^{3}$, Shereen M. Kabeel ${ }^{4}$
}

Codex : 06/2001

azhardentj@azhar.edu.eg

http://adjg.journals.ekb.eg

DOI: 10.21608/adjg.2019.7677.1088

\section{KEYWORDS}

Implant-supported prostheses, occlusion, masticatory efficiency.

\begin{abstract}
Purpose : the present study was assess the outcome of different occlusal concepts which are balanced occlusion and canine guided occlusion on the masticatory efficiency of muscles of mastication in implant supported mandibular overdentures wearers. Material and method: Ten totally edentulous patients were chosen for this study, aged 50 to 60 years old. All patients free from any systematic dieses. The patients were divided according to the treatment protocol into two groups; all patients wore denture with intra oral adjustment as a control group, (GI) five patients wore denture with bilateral balanced occlusion and (GII) five patients wore denture with canine guided occlusion. All patients were submitted to masticatory efficiency test, after one month by using different textures of foods. All data selected during the study were evaluated using ANOVA followed by Tukey's post hoc test using LSD test. $\mathrm{P}<0.05$ was considered statistically significant. Results: with soft food, there was no significant difference among the three groups, while for chewing hard type of food; the greatest mean was recorded in occlusion after oral adjustment, whereas the lowest mean was recorded in bilateral balanced occlusion. Conclusion: The two groups selected in the study, bilateral balanced occlusion as balanced concept and as a non-balanced concept canine guided occlusion both can be considered for usage effectively in implant retained overdentures without disturbing chewing efficiency.
\end{abstract}

- Paper extracted from Doctor Thesis titled "The Effect of Different Occlusal Concepts on Masticatory Efficiency in Implant Supported Mandibular Over-dentures"

1. Assistant Lecturer of Removable Prosthodontics, Faculty of Dental Medicine for Girls, Al-Azhar University.

2. Professor of Removable Prosthodontics, Vice Dean of Faculty of Dental Medicine for Girls, Al-Azhar University.

3. Professor and Head of Removable Prosthodontic Department, Faculty of Dental Medicine for Girls, Al-Azhar University.

4. Lecturer of Removable Prosthodontics, Faculty of Dental Medicine for Girls, Al-Azhar University.

*Corresponding author Email: gogo_elsadany@yahoo.com 


\section{INTRODUCTION:}

In spite of the improvements in dental healthcare and avoidance policies many patients still fail to keep their teeth due to periodontal diseases or caries or trauma. Latest updates have showed wide improvement in dental wellbeing circumstances but the rise in quality of life expectations has also improved the age and number of the elderly individuals making also the edentulous individuals in necessity need of full mouth rehabilitation. Complete dentures may introduce its own as an answer to restoring persons' oral esthetics besides mastication ability. But due to anatomical, masticatory and sensitive causes it is not all the time likely to reach the amount of stability and retention needed to offer a prosthesis that is comfy to edentulous patients especially for mandibular prosthesis wearing ${ }^{(1-4)}$.

Implant retained overdentures have been a management commonly used for the edentulous patients treatment for the last 25 years and liable to attain good proven results. Many patients particularly those who are not feeling comfort with their dentures enjoyed additional support and retention implants offer for their dentures. Implant supported overdenture has been known as the standard of care for completely edentulous patients and should be the principal choice of treatment for the edentulous mandibular $\operatorname{arch}^{(5,6)}$.

Implant occlusal overloading is frequently viewed as one of the chief causes for periimplant loss of bone and prosthesis supported by implant failure as it may lead to loss of crestal bone thus increases the periimplant diseases condition, if patients can't do post insertion clean of prosthesis and surrounding well. So an appropriate occlusal concept is necessary for long term persistence of implants particularly when a parafunctional habit is occurring. Control of implant occlusion is so important to ensure minimal and even stress distribution to the osseointegrated abutment fixtures which cannot move to compensate for possible occlusal and other technical differences so that ideal implant load and success can be achieved ${ }^{(7,8)}$.
Balanced occlusion also named balanced articulation, the bilateral, simultaneous anterior and posterior occlusal contact of teeth in centric and eccentric positions or is a concept that is largely a prosthodontic scheme which commands that maximum number of teeth should contact in all excursive positions of the mandible. This is particularly useful in complete denture production ${ }^{(9)}$.

Canine guided is demarcated as a system of mutually protected articulation in which the perpendicular and horizontal overlap of the canine teeth disengages the posterior teeth in the lateral movements of the mandible. It can be used effectively in complete dentures as it provides mandibular denture masticatory ability, retention and esthetics.Canine guided occlusion reduces the chances of tempromandibular joint dysfunction, since it reduces the lateral excursive occlusal contacts and the likelihood of interfering contacts. Accordingly the chance of muscles dysfunction is decreased ${ }^{(10,11)}$.

Masticatory function efficiency is the ability of a patient's masticatory system to decrease food types to small particles to be swallowed easy. It is a compound task affected by many aspects including mandible kinematics, occluding teeth and bite or occlusal forces, salivary activity and tongue work. For edentulous patients, masticatory efficiency is reduced because dental arches are replaced by artificial teeth. Chewing hard foods is frequently difficult for patients who wear complete dentures, and their chewing efficiency has been demonstrated to be restricted ${ }^{(12,13)}$.

THE selection of a specific occlusal concept or design for implant retained prosthesis is wide and often debatable. To date, there is no indication to recommend a precise occlusal concept for implant supported overdentures. No sufficient scientific evidence was found on relating canine guided occlusal scheme in implant-supported overdentures. Therefore, this study was directed to investigate the effects of balanced occlusion and canine guided occlusion on masticatory efficiency in implant supported overdentures ${ }^{(14)}$. 


\section{MATERIAL AND METHODS}

\section{Patient's selection:}

Ten completely edentulous patients were selected for this study aged from $50-60$, all patients have Angle class I jaw relation, free from any systemic disease that might interfere with osseointegration of implants or contraindicate surgical procedures. Residual ridges had normal morphology and free from severe bony undercuts or flappy tissue, of suitable height and width for implant placement. All patients had no abnormal habits such as bruxism or bad habits such as smoking.

The patients were informed with all procedures of our study. Only motivated patients who showed co-operation participated and an informed signed consent was assigned. Approval of REC (Research Ethic Committee) of the Faculty of Dental Medicine for Girls, AL - Azhar University was obtained.

\section{Clinical examination:}

Visual examination: Selected patients were examined intra-orally to evaluate the mucosa covering the edentulous ridge to verify the absence of any signs of inflammation, ulceration and infection. Digital examination: The width and the height of the residual ridge in the inter-foraminal region were roughly evaluated by palpating the ridge between the thumb and index fingers.

Laboratory investigation presurgical blood analysis was carried out for all patients to avoid any complications during surgery such as: the prothrombin time, complete blood picture, glycated Hemoglobin

\section{Radiographic examination:}

Cone beam radiograph was taken with a radiographic template. Availability of bone height and buccolingual width for implant placement was detected. Bone quality especially at the prospected implant sites was determined; the position of mental foramen and any bony pathological lesion and remaining root was also detected.

\section{Construction of radiographic template:}

Preliminary impression was taken for lower arch and cast was poured into stone cast. The prospective implant sites were marked on the cast at the canine regions of the lower arch. A metallic ball of $5 \mathrm{~mm}$ diameter was adapted to the marked sites using sticky wax. A wax template was then constructed on the lower ridge. The template was transferred into clear acrylic resin in the usual manner, finished, polished and checked in the patient' mouth.

\section{Complete denture construction:}

A conventional complete denture was constructed for all patients following the conventional method steps. Finished denture was examined retention, stability, extension, and articulation. Instructions were given to the patient regarding how to use the denture and how to clean it.

\section{Surgical procedures:}

The metal balls were removed from the radiographic template and holes were drilled at the proposed implant sites (canine regions) to be used as a surgical stent. All patients received two tapered, self -tapping, endosteal implants from Multisystem with $11 \mathrm{~mm}$ length and $3.2 \mathrm{~mm}$ diameter to be inserted bilaterally

\section{Flapless surgical technique:}

At first a bleeding point was created through the surgical stent, A two pilot drills 1.8 and $2.3 \mathrm{~mm}$ in diameter were used at the previously determined implant site. Then final drill was used, $2.8 \mathrm{~mm}$ to expand the site until the determined depth and width.

A paralleling rod was inserted into the osteotomy sites to ensure correct direction of both implants. The implant was taken from the inner vial by the silicon stopper, to which the implant was anchored through the placement head. Ratchet was then used to slowly screw each implant in a clockwise direction. The installation was continued until resistance was felt given an indication that crestal bone level was reached. Then the cover screws were tightened 
to the implants. After three months, second stage surgery was carried out. the cover screws were removed and the supplied metal housing with nylon liners were attached to the male ball abutment,

\section{Direct pick-up technique:}

The areas over the housings were relived from denture base with an acrylic bur till the denture is completely seated in mouth. A small amount of auto polymerizing acrylic powder and liquid was mixed and placed into dry, relieved areas within the fitting surface denture base.

The denture was placed in its housing in mouth and the patient instructed to bite light in centric occlusion. Final adjustments were done and the patient was trained for the use and care of implant and prosthesis and to have a good oral hygiene.

\section{Patient's grouping}

\section{Control group}

All ten patients wore their dentures with intra oral adjustment (just remove premature contact or any interference) as a control group for one month then were divided into two groups according to the treatment protocol then recalled to perform the masticatory efficiency test.

\section{Group I: (bilateral balanced occlusion)}

Five patients wore their dentures with changing occlusion to bilateral balanced occlusion by clinical remounting for one month then recalled to perform the masticatory efficiency test.Then the bilateral balanced occlusion was changed to canine guided occlusion by addition of autopolymerized acrylic resin in the lower canines, Then after one month the patients recalled to perform the masticatory efficiency test.

\section{Group II: (canine guided occlusion)}

Five patients wore their dentures with changing occlusion to canine guided occlusion by adding of autopolymerized acrylic resin in lower canines, in such a way that it could provide an interarch disoc- clusion space of two millimeter in the molar region during eccentric movements. Then after one month the patients recalled to perform the masticatory efficiency test. The change from the concept of canine guidance to balanced occlusion was performed by removal of the added auto polymerized acrylic resin and the bilateral balanced occlusion re-established by clinical remounting. Then after one month the patients recalled to perform the masticatory efficiency test.

\section{Clinical remounting:}

The dentures were poured into dental stone. The resultant cast was trimmed. Maxillary face bow record was carried out and transferred to articulator centric and protrusive relation records were taken by softened compound impression material to adjust condylar guided of semi adjustable articulator

\section{Evaluation of Masticatory efficiency:}

Four measurements were recorded during chewing food specimens:

1. No. of chewing strokes until the first swallow.

2. No. of chewing strokes until the patient's mouth became clear of food.

3. Time estimated in second till the first swallow.

4. Time estimated in seconds till the patient's mouth became free of food.

- Patient chewed one piece of each food for five times. The mean of the five recordings was considered the masticatory efficiency parameter for that patient.

\section{Statistical analysis}

Values were presented as mean and standard deviation (S.D) values. Data were examined for normality using Kolmogorov-Smirnov test of normality. The results of Kolmogorov-Smirnov test indicated that most of data were normally distributed (parametric data) on sequently one way analysis of variance (ANOVA) test was used to compare between groups. This was tracked by Tukey's post hoc test when the difference was found to be significant. 


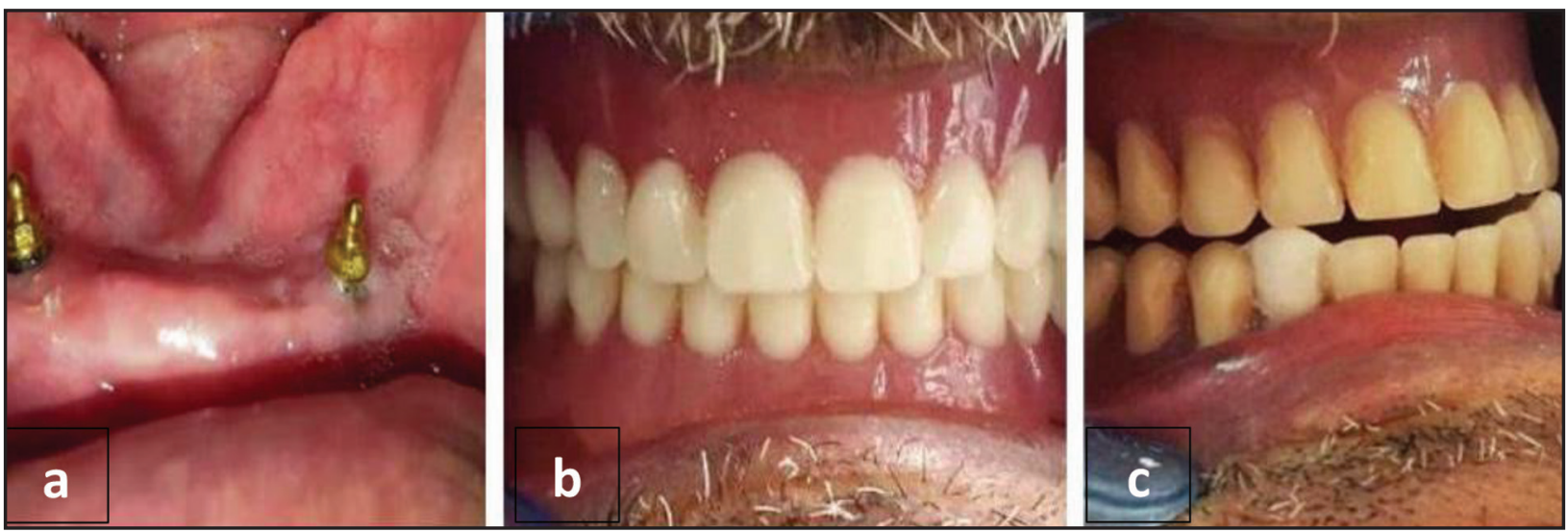

Figure(1) a- implants attachment b-bilateral balanced occlusion c-Canine guided occlusion .

The significance level was set at $\mathrm{p}<0.05$. Statistical analysis was performed with SPSS 18.0 (Statistical Package or Scientific Studies, SPSS, Inc, Chicago, IL, USA) for Windows.

\section{RESULTS}

Masticatory efficiency:

With chewing soft type of food, the highest mean value as recorded in occlusion after oral adjustment, whereas the lowest mean value was recorded in bilateral balanced occlusion. ANOVA test shown that the difference among groups was statistically non-significant regarding number of swallows $(\mathrm{P}=$
$0.082,0.051$ in first swallow and mouth clearance respectively) and time of swallows $(\mathrm{P}=0.295,0.34$ in first swallow and mouth clearance respectively), (Table 1, Fig. 2)

With chewing hard type of food, the highest mean value was recorded in occlusion after oral adjustment, whereas the lowest mean was recorded in bilateral balanced occlusion. ANOVA test revealed that the difference between groups was statistically significant regarding number of swallows $(\mathrm{P}=$ $0.049,0.048$ in first swallow and mouth clearance respectively) and time of swallows $(\mathrm{P}=0.047,0.049$ in first swallow and mouth clearance respectively), (Table 1, Fig. 2)

Table (1) Masticatory efficiency of muscles of mastication

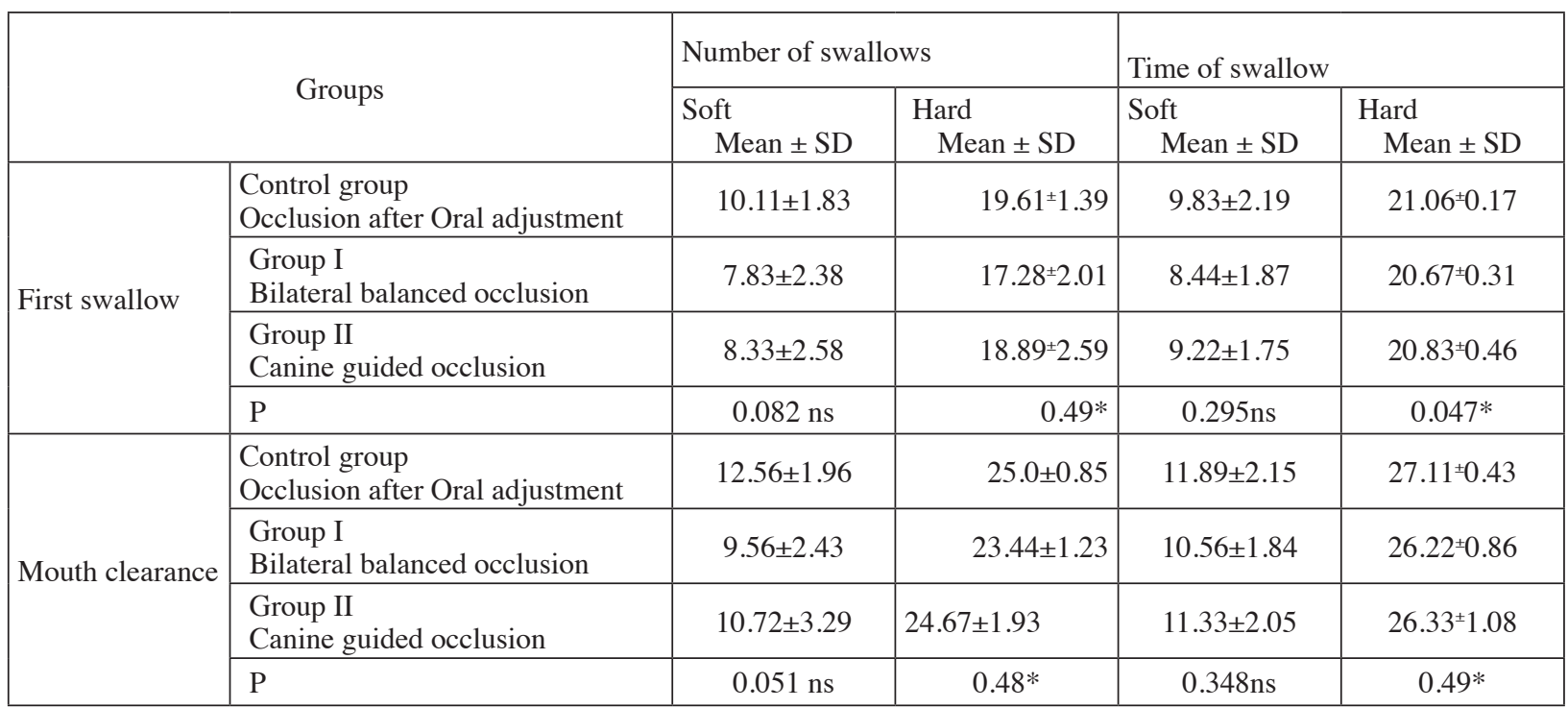




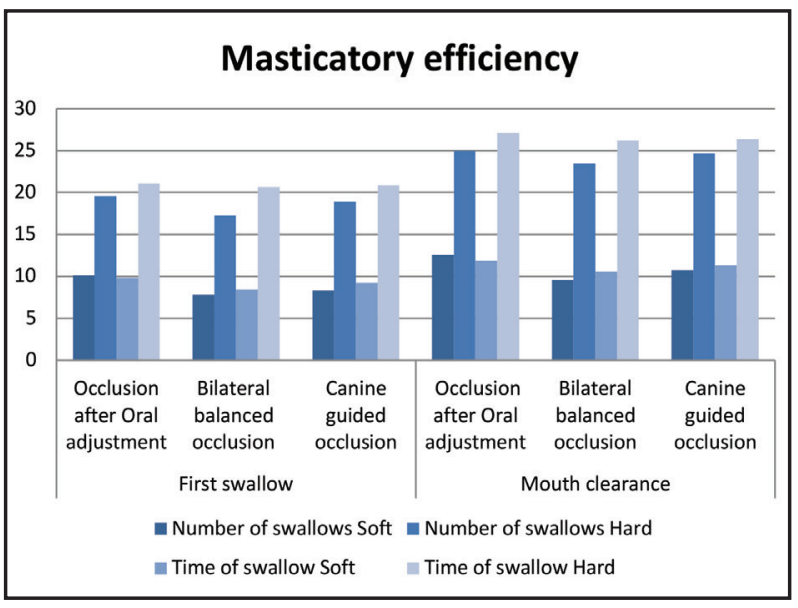

Figure (2) Bar chart showing Masticatory efficiency of muscles of mastication.

\section{DISCUSSION}

The current study was designed as a comparative clinical trial with strict inclusion and exclusion criteria to remove variables that would lead to uncertainty in the validity of data. In this study, the patients in each group received one denture with two different occlusal concepts representing balanced occlusion and canine guided occlusion. The occlusal scheme was interchanged so that all patients in this study were exposed to the two occlusal concepts for the similar periods of time.

The patients were selected with age ranging from 50- 60 years to avoid several risk factors, which may include various medical problems which reduce physical ability of older patients and physiological problems with subsequent reduction of the patient coordination. Correspondingly risk factors were related precisely to the oral system are dry mouth due to salivary hypo-function by aging, supportive structures experience a number of wellknown changes, muscle and mucosa atrophy and excessive bone loss ${ }^{(15)}$.

The patient's systemic conditions were considered in this study to avoid any metabolic changes that affect post-operative healing and remodeling. Factors that may make difficulties for dental implant placement in diabetic patients include their larger predisposition to infection and late wound curing. All uncontrolled diabetic patients were omitted from the current study due to the expected high risk of periimplantitis and higher level of implant failure ${ }^{(16)}$.

Heavy smokers were excluded from the current study as previously study have shown that smoking as a significant risk for implant predisposing cause of failure. As tobacco contains carbon monoxide, tar and hydrogen cyanide, smoking practice may cause late bone healing, poor bone quantity and quality, increased periimplantitis and also bigger bone loss rate $^{(17)}$

The Flapless surgical protocol approach which was used in this study has extended acceptance in the last few years. An understandable improvement of this technique is the exclusion of the necessity to surgically raised flap and the exposure of the underlying bone to insert the implant, increase in patient comfort and his acceptance, decrease of loss of the soft tissues that heal more faster with minimal problems, decreased pain, inflammation, surgical technique time and enhanced recovery ${ }^{(18)}$.

In an assessment of different types of test foods used for evaluating masticatory efficiency in denture wearers it was concluded that soft food like sausages, sardines and banana were between the easiest ones whereas the lettuce fibrous ones were more tough to be chewed so banana as an example of soft food and carrot as an example of hard food were chosen in this study to represent normal food varieties that patients use in their normal diet. So, they are suitable to evaluate masticatory efficiency of those patients. Types of food which was used in the study have less variability and hence they are good indicators of the difference between different occlusal concepts during mastication. The special characteristics of each food item can affect the use of one food item vs. one another and too can affect the effectiveness of masticatory system ${ }^{(19)}$.

Masticatory efficiency study performed to compare implant supported overdentures with different 
occlusal schemes comprised of bilateral balanced occlusion and canine guided occlusion that both contain coinciding contact in centric relation but they differ in eccentric movements ${ }^{(20)}$.

The results of the present study did not show any significant difference of masticatory efficiency during masticating soft type of food among the occlusal concepts involved in the study. This conclusion agrees with studies which likened the result of bilateral balanced occlusion and canine guided occlusion on masticatory efficiency in implant-retained mandibular overdentures and found no significant difference $^{(21)}$.

The results also coincide with other studies which compared masticatory efficiency and evaluation of electromyographic activity between bilateral balanced occlusion, lingualized, canine guided occlusion, and monoplane occlusal concepts and bring into being non-significant difference ${ }^{(14)}$.

These outcomes were correspondingly similar to dissimilar author who identified that bilateral balanced occlusion did not increase masticatory efficiency when likened with canine guided. Since this measures involved in the assembly of a set of dentures with bilateral balanced occlusion are greatly more complicated and time-consuming than the dentures for canine-guided occlusion for dentists. It is more complicated to attain bilateral balanced occlusion during the organization of prosthetic teeth and clinical modifications. Thus, it is practical to use technical ways that produce satisfactory clinical results in a least extent of time and effort ${ }^{(22,23)}$.

The present study displayed that masticatory efficiency improved in all patients during chewing of hard food than during chewing soft food in all groups. This was in agreement with a previous study where authors found that the number of cycles increased with chewing hard type of food rather than with the soft type of food and the time taken by the patients to form a bolus of food which is ready to be swallowed increased in case of hard food than soft food ${ }^{(22)}$.

\section{CONCLUSION}

Within the limits of this study of mandibular implant-supported overdenture, both bilateral balanced occlusion and canine guided occlusion can be used effectively in implant supported overdentures with no any significant effect on masticatory function.

\section{REFERENCES}

1. Sonego MV, Goiato MC, Santos DM. Electromyography evaluation of masseter and temporalis, bite force, and quality of life in elderly patients during the adaptation of mandibular implant-supported overdentures. Clin Oral Impl Res J 2016; 43: 1-6.

2. Awad MA, Rashid F, Feine JS. The effect of mandibular 2-implant overdentures on oral health-related quality of life: an international multicenter study. Clin Oral Impl Res 2014; 25: $46-51$.

3. Dakhilalian M, Rismanchian M, Fazel A, Basiri K. Conventional versus implant- retained overlay dentures: a pilot study of masseter and anterior temporalis electromyography. J Oral Impl 2014; 40: 418- 24.

4. Kuoppala R, Raustia A. Preliminary observations regarding treatment outcomes in patients treated with maxillary implant overdentures in a university clinic. Int $\mathrm{J}$ Prosthodont 2015; 28: 637-40.

5. Gracht I, Derks A, Haselhuhn K. EMG correlations of edentulous patients with implant overdentures and fixed dental prostheses compared to conventional complete dentures and dentates; a systematic review and metaanalysis. Clin Oral Impl Res J 2017; 28:765-73.

6. Maheshwari R, Hans S, Mittal S, Aneja S, Duhan J. Implant supported mandibular overdenture with bilateral balanced occlusion. J Oral Health Comm Dent 2015; 9: 44-6.

7. Verma M, Nanda A, Sood A. Principles of occlusion in implant dentistry. J Int Clin Dent Res Organ, 2015; 7: 27-33.

8. Kim Y,Oh TJ, Misch CE, Wang HL. Occlusal considerations in implant therapy: Clinical guidelines with biomechanical rationale. Clin Oral Impl Res 2005; 16: 26-35.

9. Pregl V, Pošti SD. Influence of balanced occlusion in complete dentures on the decrease in the reduction of an edentulous ridge. J Prosthet Dent 2012; 69: 155- 60. 
10. Valenzuela S, Portus C, Miralles R. Bilateral supra- and infrahyoid EMG activity during eccentric jaw clenching and tooth grinding tasks in subjects with canine guided or group function. J Cranio 2012; 30: 209- 17.

11. Pasricha N, Sidana V, Bhasin S, Makkar M. Canine protected occlusion. Indi J Oral Sci 2012; 3:13- 8.

12. Magalha IB, Pereira LJ, Marques IS, Gameiro GH. The influence of malocclusion on masticatory performance. Angle Orthodontist J 2010; 80: 5.

13. Karbownik AM, Chladek G, Żmudzki J, Kasperski J. Chewing efficiency and occlusal forces in PMMA, acetal and polyamide removable partial denture wearers. J Acta of Biomech 2016; 18: 138-44.

14. Neto FA, Mestriner JW, Carreiro AF. Masticatory efficiency in denture wearers with bilateral balanced occlusion and canine guided. Braz Dent J 2010; 21: 165- 9.

15. Palinkas M, Nassar M, Cecílio F, Siéssere S, Semprini M, Machado J. Age and gender influence on maximal bite force and masticatory muscles thickness. Arch Oral Biol 2010; 55: 797- 802 .

16. Naujokat H, Kunzendorf B, Wiltfang J. Dental implants and diabetes mellitus systematic review. Int J Imp Dent 2016; $2: 5-8$

17. Twito D, Sade P. The effect of cigarette smoking habits on the outcome of dental implant treatment. Peer J 2014; 2: $546-9$
18. Nidhin R, Gilsan K Vasunni GK, Ajay O,Biji Kurien. Comparative evaluation of crestal bone levels following implant placement with flap and flapless techniques in posterior edentulous areas of the mandible- an in vivo study. J Dent Med Sci 2014; 13: 95-9.

19. Moynihan P, Thomason M, Walls a, Donald KG, Ghanem H. Researching the impact of oral health on diet and nutritional status: Methodological issues. J Dent 2009; 37 : 237-49.

20. Aziz HM. the effect of occlusal design on muscle activity in implant supported mandibular overdentures. CDJ 2008; 24: 335: 50.

21. Abdelhamid AM, Assaad N, Weheda AI. Evaluation of mucosal displacement and denture settlement for direct vs. indirect attachment incorporation in implant assisted mandibular overdentures. J Dent Health Oral Disord 2016, 5: $1-8$.

22. El-Okel AB. The effect of different occlusal schemes on the electromyographic activity and masticatory efficiency in complete denture wearers. Ph.D. Thesis, Department of Removable Prosthodontics, Faculty of Dentistry, Al-Azhar University. Egypt2006.

23. Kezhao O, Qing MX. Occlusal designs on masticatory ability and patient satisfaction with complete denture: A systematic review. J Dent 2013; 41: 1036- 42. 